\title{
A New Model for Simulations of Decision-Making in Management: an Expert-Psychological Approach
}

\author{
Tatyana V. Karlova ${ }^{1, *}$, Marianna V. Mikhaylova ${ }^{2}$, Natalia M. Kuznetsova ${ }^{1}$, Aleksandr Yu. Bekmeshov ${ }^{3}$, Denis V. Poltoranov \\ and Elena V. Obukhova ${ }^{1}$ \\ ${ }^{1}$ Moscow State Technological University "STANKIN", Systems and Information Processing Department, RU-127055, Moscow, Russia \\ ${ }^{2}$ Moscow State Technological University "STANKIN", Department of philosophy, RU-127055, Moscow, Russia \\ ${ }^{3}$ Institute For Design-Technological Informatics RAS, RU- 127055, Moscow, Russia
}

\begin{abstract}
The article is dedicated to the development of structural model of the influence the human factor based on research of engineering-psychological features in management decision making processes in the complex integrated automated control systems. In the real hierarchic control systems the main problem is to find the ways of structures activity by vertical and horizontal directions. The algorithm of forming the functional system activity realizes the processes in structure of human nervous system. The algorithm is created by union motivation, goal, personal meanings and the image of the result. The algorithm realizes special mental functions. Integral development of human activity abilities is giftedness contained in the union, that could be detected by functional system features realized through the human mental functions. Management making decision process program contains not only the conditions of choice of the intellectual operations but the time modes. The program envelopes the structural blocks of all activity system. The processes of program creation and management making decision provided by each of abilities in view of the function ability system's union direction simultaneously become subordinated according to its motivation activity. Information security aspects are considered in the article, particularly, the application of the complex approach to solving the protection of the functional system activity problem is proposed. For the diagnostics of the professional expert abilities the development of the methodology is needed. The methodology helps to investigate the field activity problem from the position of interdisciplinary approach to the complex technical objects based on integrated automated control systems.
\end{abstract}

\section{Introduction}

In the real hierarchical control systems the main problem is finding the ways of interconnection of the activities of structures both vertically and horizontally.

The need to model structures with arbitrary relationship arises in solving many problems.

When solving the problems of decision-making in management, it is necessary to define a formula that will link the goal with the means and mechanisms to achieve it.

In addition, there were considered the issues of identifying interdependent strategic links between information processing activities and expert-professional and psychological approach to the obtained data. This approach is a complement to the theoretical justification of the general theory of stress psychology.

As a result of the solution will be such concept as:

- efficiency indicator;

- optimality criteria;

- target function.

On the basis of the hypotheses and the creation of simulation models, it is possible to explore possible options for decision-making in management.

\section{Information base of activity}

Modern industrial enterprises are complex hierarchical systems containing a variety of structures: personnel, raw, material, energy, financial and economic resources.

Plant's operational efficiency depends on information flow rate, which provide continuous updating of production processes. The plant's operational efficiency depends on the rate of information flows, which provide continuous updating of production processes. Maintaining a holistic view of technological production and financial processes which are difficult to formally describe with a non-verbal component, based on experienced intuitive intellectual assessment of human activity, remains one of the important automation tasks.

Risk of making irrational decisions is based on the current multidisciplinary tasks' dependence on a significant number of internal and external factors.

One of the most important internal risk factors is that plant management urge on the simplification of the difficult situations and neglect expert opinion, which are at different levels of management and are associated with 
different production and professional units, and make decisions based on simplified approaches.

Automation of processes for collecting and processing expert information is not only the task of accumulation intellectual potential but also one of the most important conditions to increase the efficiency of the industrial production management system.

In general, any activity is carried out through the functioning of abilities, which, in turn, are developed through an identical attitude to goals, motives and personal meanings.

It is necessary to translate the received integrated representations based on methods of the system analysis from the verbal description into the formal one.

Formalization of verbal description, understanding of modern functions and obtaining the presented results are the main components of all stages in modeling the developing complex system.

The main part of the research is analyzing the influence of the human factor on the management processes of integrated automated systems, based on the analysis of psychological engineering aspects and investigation of the facts, which require special expert adaptation in the processes of making decisions in management.

Thus, the ability to combine the data of the three parameters (components) in the framework of joint work of the system realizes the activity.

According to the goal, based on sequentially performed human mental functions the program of operations tools choice is executed. In other words the fulfilment of intellectual operations with simultaneously beginning in interaction mode.

Information base of activity is being formed by means of perception and memory, while the program design, which performs the practical action realization, is being formed by means of thinking process.

The obtained results and their parameters is the basis of the plan for practical actions with the subsequent correction of the program as a whole and as separate psychological functions.

Programs' interdependency is the most important factor of action system genesis.

The program of making decision process contains not only the choice of conditions in the performing of intellectual operations, but also time modes, and includes structural blocks of all action system.

The main choice condition of any management decision is generating criteria for goal achievement and objective.

Algorithm of forming the function activity system performs the processes in human nervous system structure. It is created by means of union motivation, goal, personal meaning and type of the obtained result. The algorithm realizes the specified psychological functions.

The integral ability of a person, manifested in the process of activity, is giftedness, which consists of the unity, determined by the properties of functional systems that implement the mental functions of a person.

\section{Expert judgement method}

The interaction ability problem is complicated, as during its solving by means of expert judgement method, the consistency of opinions is demanded. Also the using of rank correlation theory methods is needed.

Consistency degree of experts' meanings is concordance factor:

$$
W=\frac{12 d}{m^{2}\left(n^{3}-n\right)}
$$

where: $d=\sum_{i=1}^{n} d_{i}^{2}=\sum_{i=1}^{n}\left[\sum_{i=2}^{m} r_{i j}-0,5 m(n+1)\right]^{2}$;

$m$ - expert number;

$j=1, \ldots, m, n-$ number of considered properties;

$i=1, \ldots, n, r_{i j}$ - order number, occupied by $i$-property in range of $j$-expert;

$d_{i}$ - change the rang sum of i-property from the normal rang sum of $n$ properties.

The value of concordance factors $W$ provides an opportunity to assess the consistency of the preferred solution, given by each expert.

Limiting values $W$ are found in domain $0 \leq W \leq 1$.

In the case when $\mathrm{W}=0$, it is a total opposition of opinions.

In the case where $\mathrm{W}=1$, there is a full matching of ranges. In practice, it means that credibility value of expert judgment is considerable.

Personal experts' motivation is included in the functional systems of each ability. Thus the uniform operational orientation of all abilities manifestation has to be provided.

Program creation and making decision processes, which are provided by each of ability according to union direction of system functionality, simultaneously become correlated with the motivation activity.

Result is the formation of functional giftedness metasystem.

Each ability is displayed in the metasystem not only by natural properties but also by number of acquired manifestation.

Qualitative direction of specific activity for concrete ability becomes a singular vertex of giftedness and could be the system quality.

It is important to consider such aspects as:

- actions structure relations to the structure of activity;

- $\quad$ the structures' relationship;

- actions' structure and activities with it's subject's abilities correlation;

realization mechanisms.

Directing vector of activity is the motive and the goal simultaneously, and the action vector is the subgoal, which is correlated with the objective achievement.

Any action has the special place in the general structure activity.

Specific actions could be combined by means of motive and goal to the action where each of the action subsystem components has it's own special contents and is integrated by the motivation and goal to united 
structure (Fig. 1).

The special actions' subsystem components are integrated to the complex system according to contents. The system contains the blocks of psychological activities. The making decision block receives the information from operational mechanisms' forming, intellectual operations, expert parameters correction blocks.

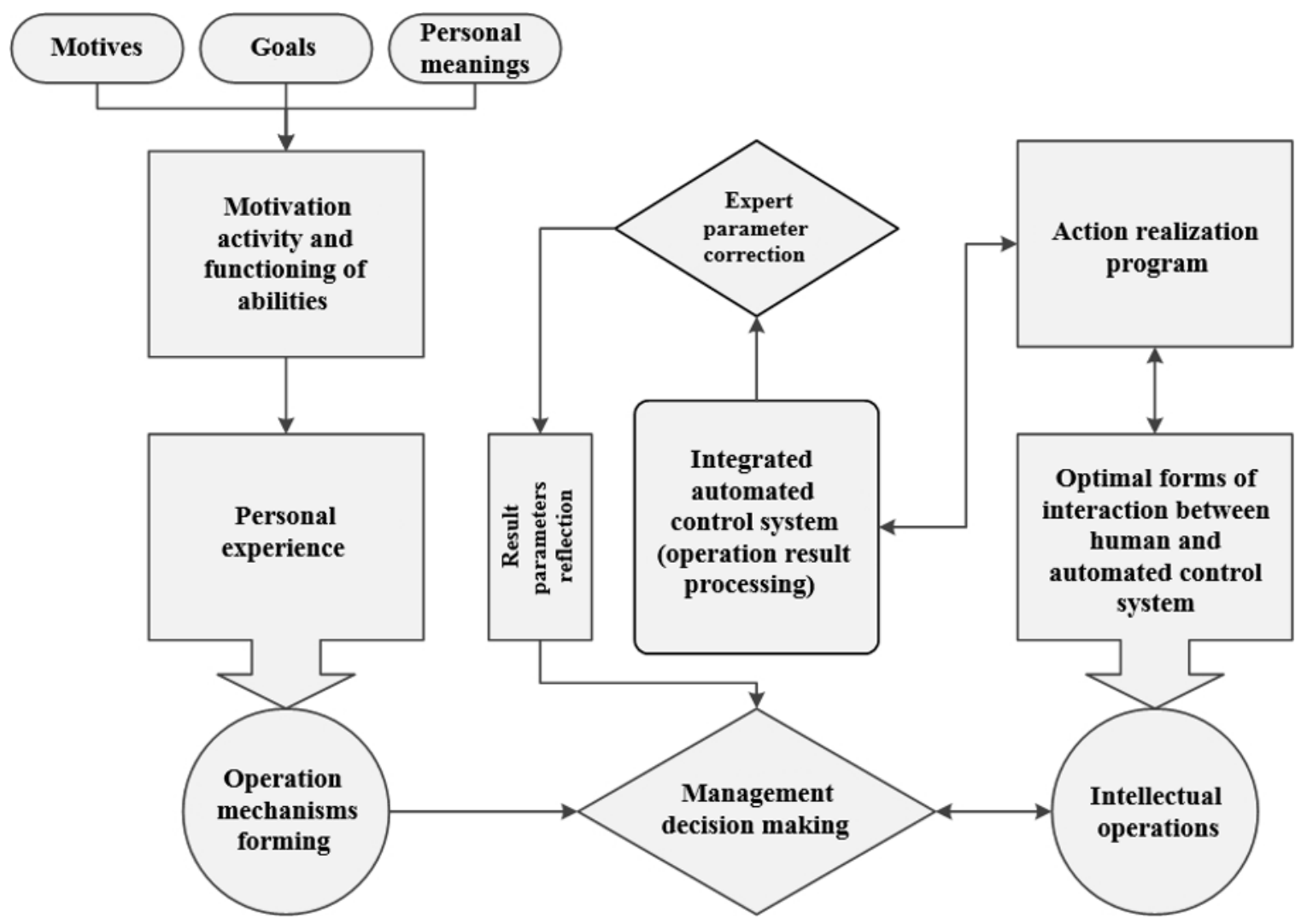

Fig. 1. Functional system of experts’ abilities.

Each psychical action includes the special non-verbal abilities, such as perception, thinking, imagination, memory, sensory motorics. [10]

Abilities interaction in the general functional system of activity are shown on Fig. 2.

The abilities could be the mechanisms, which realize the activity.

In its turn the abilities are attracted to the activity for its demand. Simultaneously in process of its activity the abilities are being developed. And the more complicated activity is, the faster development takes place.

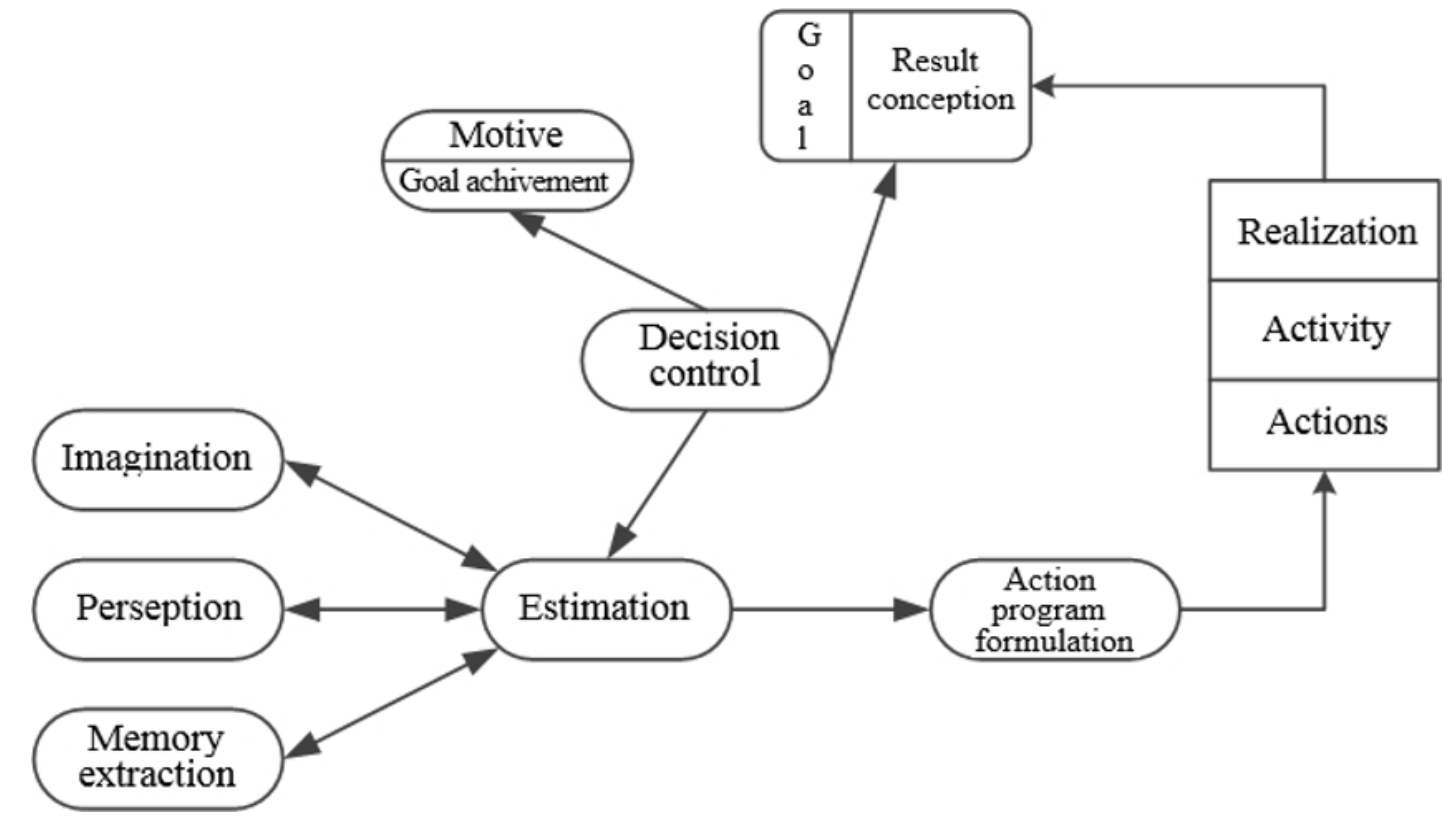

Fig. 2. The interaction of abilities in a generalized functional system of activity. 


\section{Information security aspects}

When making management decisions it is important to bear in mind the information defence principles.

The functional activity system constantly cooperates with external environment, by means of which the attacks could be realized.

The most dangerous are «zero day attacks» and APT advanced persistent threats.

Unfortunately, there are no any methods for fighting with «zero day attacks» since the technologies and algorithms of the attacks are absolutely new for the defence systems.

Today APTs are the most dangerous attacks since they are long-term and, as a consequence, are difficult to research and classify. [3]

For the functional activity system to defence from the described attacks the complex approach must be used. The approach includes a number of security measures:

- soft and hardware defence methods (e.g. DLP - Data Leak Prevention systems);

- technical defence methods (e.g. application special methods while designing and buildings the constructions);

- cryptographic protection (as symmetric as asymmetric cipher algorithms);

- organization defence methods (the developing of normal exploitation base). [8]

The most vulnerable places are «exit points» from functional activity system - the interface with external environment. Exactly through the interface there is the main information traffic, which simultaneously contains strategic important data for making decisions and potentially dangerous information sequences. [9]

The scheme of interaction between the functional activity system and the environment is shown on Fig. 3.



Fig. 3. The interaction between the functional activity system and the environment.

It is important to remember that only the complex approach to the information defence could supply the maximum security level. [4-7]

\section{Conclusion}

For diagnostics of professional-expert abilities the development of the principles is needed. The principles should cooperate the problem field research from the position of interdisciplinary approach to the complex technical objects based on integrated automated control system.

The analysis of production systems is made on the basis of the accepted structure of hierarchical subordination using the object approach.

It is important to note that for making correct decision and to reduce the risks the system should not be completely automatic - the main control should be in the human hands. The automated system should be advisory in nature, and the responsibility for decisions is borne by the person himself.

This work was carried out using equipment provided by the Center of Collective Use of MSUT "STANKIN".

\section{References}

1. Actual problems in the labour psychology, engineer psychology and ergonomics. Vol. 2 / Redaction V.A. Bodrova, L.A. Zhuravleva (M.: publishing house «Psyhology Institution RAS», 2011) ISBN: 978-5-9270-0182-8

2. J. Klir, Systemology. Automation solutions of system tasks: translate from English (M. Radio and communication, 1990)

3. T.V. Karlova, A.Y. Bekmeshov, S.A. Sheptunov, N.M. Kuznetsova, 2016 IEEE Conference on Quality Management, Transport and Information Security, Information Technologies, 72-76 (2016) ISBN: 978-1-5090-3681-3

4. Masaaki Imai. Japan Management Quality Methods School. [Electronic resource]: http://quality.eup.ru

5. European quality management experience. [Electronic resource]: www.9001-2001.ru

6. Quality Management in the USA. [Electronic resource]: www.9001-2001.ru

7. Steve McConnell, Code complete / Second Edition (Microsoft Press, 2004)

8. O.O. Martynov, Scientific and Technical Journal "Engine" (Moscow: LLC "Editorial Board of the magazine "Engines"), 56-57 (2012)

9. T.V. Karlova, N.M. Kuznetsova, Scientific and technical journal "Bulletin of the Bryansk state technical University" 4(57), 84-89 (2017) DOI: 10.12737/issue_5a02f9f7150658.06648590

10. T.V. Karlova, A.Yu. Bekmeshov, M.V. Mikhailova, A.N. Zapolskaya, Scientific and technical journal "Bulletin of the Bryansk state technical University" 2(55), 191-195 (2017) ISSN 1999-8775 